\title{
The Absence of Eukaryotic Initiation Factor elF(iso)4E Affects the Systemic Spread of a Tobacco etch virus Isolate in Arabidopsis thaliana
}

\author{
Carlos A. Contreras-Paredes, ${ }^{1}$ Laura Silva-Rosales, ${ }^{2}$ José-Antonio Daròs, ${ }^{3}$ Naholi D. Alejandri-Ramírez, \\ and Tzvetanka D. Dinkova ${ }^{1}$ \\ ${ }^{1}$ Departamento de Bioquímica, Facultad de Química, Universidad Nacional Autónoma de México, 04510, México, D. F., \\ México; ${ }^{2}$ Departamento de Ingeniería Genética, Cinvestav-Irapuato, Km 9.6 Lib. Nte. Carr. Irapuato-León, Irapuato, Gto. \\ 36821 México; ${ }^{3}$ Instituto de Biología Molecular y Celular de Plantas (Consejo Superior de Investigaciones Científicas- \\ Universidad Politécnica de Valencia), Avenida de los Naranjos, 46022 Valencia, Spain
}

Submitted 9 September 2012. Accepted 4 December 2012.

Translation initiation factor eIF4E exerts an important role during infection of viral species in the family Potyviridae. Particularly, a eIF(iso)4E family member is required for Arabidopsis thaliana susceptibility to Turnip mosaic virus, Lettuce mosaic virus, and Tobacco etch virus (TEV). In addition, a resistance mechanism named restriction of TEV movement (RTM) in A. thaliana controls the systemic spread of TEV in Col-0 ecotype. Here, we describe that TEV-TAMPS, a Mexican isolate, overcomes the RTM resistance mechanism reported for TEV-7DA infection of the Col-0 ecotype but depends on eIF(iso)4E for its systemic spread. To understand at which level eIF(iso)4E participates in $A$. thaliana TEV-TAMPS infection, the viral RNA replication and translation were measured. The absence or overexpression of eIF(iso) $4 \mathrm{E}$ did not affect viral translation, and replication was still observed in the absence of eIF(iso)4E. However, the TEV-TAMPS systemic spread was completely abolished in the null mutant. The viral protein genome-linked (VPg) precursor NIa was found in coimmunoprecipitated complexes with both, eIF(iso)4E and eIF4E. However, the viral coat protein (CP) was only present in the eIF(iso)4E complexes. Since both the VPg and the CP proteins are needed for systemic spread, we propose a role of $A$. thaliana eIF(iso) $4 \mathrm{E}$ in the movement of TEVTAMPS within this host.

Successful colonization of a plant host by viruses is mediated through molecular interactions between the host and viral factors. Such interactions mediate translation and replication of the viral genome, invasion of the host by cell-to-cell movement through plasmodesmata, and long-distance movement through the vascular system (Carrington and Whitham 1998; Taliansky et al. 2008).

During the past decade, a central role of the translation initiation factor eIF4E has become evident during infection of viral species in the family Potyviridae. Point mutations or polymor-

Corresponding author: T. D. Dinkova; Telephone: +5255 56225277; Fax: +52 55 56225329; E-mail: cesy@unam.mx

* The $\boldsymbol{e}$-Xtra logo stands for "electronic extra" and indicates that three supplementary figures and one supplementary table are published online.

() 2013 The American Phytopathological Society phisms within eIF4E family members have been identified as the products of recessive resistance genes and are considered as markers for viral resistance in a broad spectrum of hosts and potyviruses (Ashby et al. 2011; Gao et al. 2004; Kang et al. 2005; Nicaise et al. 2007; Robaglia and Caranta 2006). Such mutations usually do not affect the endogenous cellular role of eIF4E. This protein is involved in translation initiation of capped mRNAs by directly binding the Cap structure, $\mathrm{m}^{7} \mathrm{GpppN}$ (where $\mathrm{N}$ is any nucleotide), at the $5^{\prime}$ end of the mRNA through $\pi$ - $\pi$ stacking interactions as well as to the eIF4G protein to form the eIF4F complex (Jackson et al. 2010). In plants, three members of the eIF4E family have been characterized: eIF4E, eIF(iso)4E, and nCBP (Browning 2004). eIF4E forms a complex with eIF4G named eIF4F, whereas eIF(iso)4E binds to eIF(iso)4G to form the eIF(iso)4F complex. The expression of each member of the eIF4E family is apparently under differential regulatory mechanisms depending upon specific tissue and developmental stages (Dinkova et al. 2000; Rodriguez et al. 1998; Ruud et al. 1998). Particularly, eIF4E and eIF(iso)4E have been more broadly studied and have shown both redundant and specific roles as part of the eIF4F and eIF(iso)4F complexes during in vitro and in vivo translation of cellular mRNAs (Dinkova et al. 2011; Gallie 2001; Martinez-Silva et al. 2012).

Natural and insertional mutants in the eIF4E family have been studied in Arabidopsis thaliana (Duprat et al. 2002; Hwang et al. 2009; Jenner et al. 2010; Sato et al. 2005), pepper and tomato (Kang et al. 2005; Ruffel et al. 2002, 2005), pea (Gao et al. 2004), lettuce (Nicaise et al. 2003), and common bean (Naderpour et al. 2010). The mechanisms by which eIF4E proteins are involved in potyvirus infection are not completely understood. However, it has been shown that they interact with the viral genome-linked protein (VPg) bound to the $5^{\prime}$ end of the viral genome. It has been proposed that inhibition of cellular mRNA translation might be achieved by the competition between VPg and Cap for a particular eIF4E factor (Khan et al. 2008; Leonard et al. 2000). Mutant alleles of eIF4E showing impairment of the interaction with VPg are responsible for observed resistances to potyviral infections (Gao et al. 2004; Lellis et al. 2002). The central region of the VPg has been implicated in the interaction with eIF4E (Roudet-Tavert et al. 2007), and the VPg protein itself has been proposed as an avirulence factor in the potyvirus-plant interaction (Rajamaki and Valkonen 1999).

Other host resistance factors different from eIF4E have also been documented. Some of them act at different steps of the 
viral infection such as short- or long-distance movement. For example, the restriction of Tobacco etch virus (TEV) movement (RTM) described in A. thaliana controls the systemic spread of TEV, Plum pox virus (PPV), and Lettuce mosaic virus (LMV) (Decroocq et al. 2006, 2009; Whitham et al. 1999). TEV belongs to the family Potyviridae and infects a broad range of host plants. Its genome is composed of a positive singlestranded RNA of about $10 \mathrm{~kb}$ with a VPg covalently attached to the $5^{\prime}$ end and a poly(A) tail at the $3^{\prime}$ end. The viral genome contains a long open reading frame coding for about 10 mature viral proteins (Urcuqui-Inchima et al. 2001) and a small open reading frame called PIPO, which is inserted in the P3 sequence and produces an additional protein (P3N-PIPO) that acts as a cell-to-cell movement protein (Chung et al. 2008; Wei et al. 2010).

The first experiments describing the RTM mechanism were performed using an isolate named TEV-7DA. The RTM response requires at least three loci, RTM1, RTM2, and RTM3. $R T M 1$ encodes a protein belonging to the jacalin family, which is involved in defense against insects and fungi (Chisholm et al. 2000). The RTM2 locus corresponds to a protein with a trans membrane domain similar to small heat-shock proteins. Its expression is not heat-inducible and, apparently, is not involved in a thermo-tolerance mechanism (Whitham et al. 2000). RTM1 and RTM2 associate with the phloem, perhaps within the sieve elements, to restrict TEV long-distance movement (Chisholm et al. 2001). A third RTM loci (RTM3) was characterized (Cosson et al. 2010). It encodes a protein be-



Fig. 1. Arabidopsis thaliana infection with a Mexican Tobacco etch virus (TEV) isolate (TEV-TAMPS). A, The TEV-TAMPS RNA was detected by final point reverse transcription-polymerase chain reaction in local (L) and systemic (S) leaves of wild-type Col-0 ecotype (Col-0), AteIF(iso)4E-1 knockout mutant ([iso]4E1], AteIF(iso)4E-2 overexpressing mutant ([iso] $4 E 2$ ), and wild-type C24 ecotype (C24) after inoculation (0L) and at 4 or 15 days postinoculation (4L, 4S, and $15 \mathrm{~S})$. The viral RNA was present in systemic leaves of Col-0, (iso) $4 E 2$, and C24 wild-type plants at 4 and 15 days postinoculation but was undetectable in systemic tissues of the (iso) 4E1 knockout mutant. Mock = leaves inoculated with virus-free inoculation buffer; $e E F 1 \alpha=$ the elongation factor $1 \alpha$, which was used as an internal control; RT $(-)=$ reaction without reverse transcriptase. $\mathbf{B}$, The percentage of $A$. thaliana Col-0 plants systemically infected with TEVTAMPS or TEV-7DA isolates. Viral presence was measured with enzymelinked immunosorbent assay against TEV coat protein. longing to an undefined family of 29 members with Meprin and TRAF homology domains. Recently, two additional RTM genes have been identified, indicating that the nonconventional RTM resistance is a complex biological process (Cosson et al. 2012). At present, it is not clear how the RTM proteins restrict long-distance movement of potyviruses and whether they interact with viral components. However, a possible regulation by plant hormones opens new venues of virus resistance regulation (Cosson et al. 2012). A connection between RTM and eIF4E family member-associated resistance has not been reported to date.

In A. thaliana, eIF(iso)4E but not eIF4E is required for successful viral colonization in the case of TEV (Lellis et al. 2002) and other potyviruses, such as Turnip mosaic virus (TuMV) and LMV (Duprat et al. 2002). At least three potential roles have been considered for the VPg-eIF(iso)4E interactions during TEV infection: i) a function in viral RNA translation based on the eIF(iso)4E role in translation initiation, ii) protection of the viral mRNA $5^{\prime}$ end against enzymatic degradation through the eIF(iso)4E bound to $\mathrm{VPg}$, and iii) appropriate intracellular localization and transport of the potyviral RNA as part of ribonucleoproteic complexes through interaction with cellular microtubules (Lellis et al. 2002; Okade et al. 2009).

Here, we assayed the infectivity of a Mexican isolate of TEV (TEV-TAMPS) in A. thaliana plants (Col-0 ecotype). Curiously, we found that this isolate was able to overcome the RTM resistance mechanism reported for the Col-0 ecotype as compared with TEV-7DA, which is susceptible to RTM. However, when using eIF(iso) $4 E$ knockout plants, no systemic spread of TEV-TAMPS could be observed. The finding that TEV-TAMPS was able to overcome RTM only in the presence of eIF(iso)4E prompted us to investigate whether eIF(iso) $4 \mathrm{E}$ was involved in the systemic spread of this isolate or in either viral translation, replication, or both. After sequencing the TEV-TAMPS VPg and coat protein (CP), we found particular amino acid changes in variable regions of these proteins, as compared with other TEV isolates deposited in the GeneBank database, mostly from the Americas (Allison et al. 1985; Chu et al. 1995) but one from Asia. Translation of TEV-TAMPS was not importantly affected by the absence or overexpression of eIF(iso)4E. However, the presence of eIF(iso)4E was required for the TEV-TAMPS systemic spread in A. thaliana. Finally, although the VPg precursor NIa was found in vivo in coimmunoprecipitated complexes with both eIF(iso)4E and eIF4E, the viral CP was only present in the eIF(iso)4E complexes. Since both the VPg and the CP proteins are needed for the systemic viral movement, we propose that $\mathrm{IF}$ (iso)4E is required for the systemic spread of TEV-TAMPS.

\section{RESULTS}

A Mexican TEV isolate is able to overcome the RTM resistance mechanism of an A. thaliana Col-0 ecotype.

The RTM resistance mechanism reported for $A$. thaliana Col-0 ecotype affects the systemic spread of TEV, LMV, and PPV (Chisholm et al. 2001; Cosson et al. 2010; Mahajan et al. 1998). The viral isolates tested in these reports moved cell-tocell in inoculated leaves but did not move systemically. For the same viruses, the cap-binding protein eIF(iso)4E has been identified as a factor required for successful infection (Robaglia and Caranta 2006).

Here, we tested whether a Mexican TEV isolate (TEVTAMPS) was able to infect an A. thaliana Col-0 ecotype, previously reported to have the RTM resistance mechanism, as compared with the C24 ecotype. Additionally, eIF(iso) $4 E$ knockout mutant plants in the Col-0 ecotype background were tested to find at which level this factor is required in the viral 
infection. The TEV-TAMPS viral RNA was detected by reverse transcription-polymerase chain reaction (RT-PCR) at 4 days after inoculation (dpi) on either local or systemic leaves of wild-type Col-0 and C24 plants (Fig. 1A). At 15 dpi, TEVTAMPS was detected at similar levels in the systemic leaves of both ecotypes. Interestingly, the TEV-TAMPS RNA was not detected in the systemic leaves of AteIF(iso)4E-1 mutant ([iso] $4 E-1)$, previously reported as resistant to potyvirus infection (Duprat et al. 2002).

The ability of TEV-TAMPS to systemically spread in Col-0 wild-type (WT) plants was compared with TEV-7DA at 15 dpi, using CP quantitation by enzyme-linked immunosorbent assay (ELISA) (Fig. 1B). This experiment was performed with 20 plants per isolate in three independent experiments. Each plant was tested in duplicate technical replicate. The viral $\mathrm{CP}$ was detected in systemic leaves of only two from the $20 \mathrm{TEV}$ 7DA-inoculated plants, whereas 19 of 20 of the TEVTAMPS-inoculated plants gave systemically positive signal. These observations indicated that TEV-TAMPS but not TEV7DA was able to overcome the RTM resistance mechanism reported for A. thaliana Col-0 (Mahajan et al. 1998). Although the molecular basis of RTM has not yet been discovered and the particular viral targets for this host defense mechanism are not known, several evidences have shown that the viral NIa protein and the CP are necessary for systemic spread of the virus (Dolja et al. 1995; Urcuqui-Inchima et al. 2001). Hence, amino acid changes in these proteins could be associated with the RTM overcome in A. thaliana, as has been shown for the PPV CP amino terminal region (Decroocq et al. 2009).

\section{Amino acid differences within VPg and CP of TEV-7DA and TEV-TAMPS isolates in the context of other TEV isolates.}

To find out whether TEV-TAMPS carries particular mutations as compared with those reported for TEV-7DA, the regions corresponding to these two proteins were sequenced. The TEV-TAMPS VPg and CP (GenBank accessions JX512812_ MX_TAMPS and JX512813_MX_TAMPS, respectively) were aligned with sequences from TEV isolates available at the National Center for Biotechnology Information (NCBI) database, including accessions reported as unable to spread systemically in the $A$. thaliana Col-0 ecotype, such as TEV-7DA (US_7DA_DQ986288), TEV-HAT (US_HAT_M11458), US_ M15239, US_NW_L38714, and VE_CT8_EU334787, as well as isolates for which the ability of systemic movement in $A$. thaliana has not been described. Several amino acid substitutions were found, particularly in a variable core region and in a less variable one at the carboxy terminus of the VPg sequence (Supplementary Fig. S1). These substitutions lie outside the region reported to interact with $\mathrm{eIF}$ (iso)4E or the viral RNA. The alignment of $\mathrm{CP}$ sequences showed high variability, particularly at the TEV-TAMPS amino-terminal region near to the DAG motif needed for aphid transmission (Supplementary Fig. S2), as has been shown for other potyviruses (Rybicki and Shukla 1992; Silva-Rosales et al. 2000).

Using the above alignments, the evolutionary relatedness between TEV-TAMPS and other TEV isolates affected by the RTM mechanism was inferred from phylogenetic analysis using the Geneious alignment and tree builder, with the JukesCantor genetic distance model and the neighbor-joining method. The phylogenetic tree based on the VPg sequence showed separated groups for isolates from two countries in North America (the United States and Mexico) and one in South America (Venezuela) (Fig. 2A). Viral isolates affected by the RTM resistance mechanism (US_7DA_DQ986288 and $U S_{-}$ HAT_M11458) grouped together. TEV-TAMPS (MX_TAMPS_ $J X 512812$ ), overcoming the RTM resistance mechanism, stood alone on a separate branch of the other Mexican isolates, whose ability to spread systemically in the A. thaliana Col-0 ecotype is not known. The same applies for the Venezuelan isolates that form another group.


Fig. 2. Phylogenetic relationship of Tobacco etch virus (TEV) isolates based on the amino acid sequences of $\mathbf{A}$, viral genome-linked protein (VPg) and B, coat protein (CP). The TEV-TAMPS VPg branch (MX_TAMPS JX512812) was distant from the group of TEV-7DA, and TEV-HAT isolates reported as susceptible to the Arabidopsis thaliana Col-0 restriction of Tobacco etch virus (TEV) movement mechanism (US_7DA_ DQ986288 and US_HAT_M11458) and, curiously, from other Mexican isolates grouped together as well. According to the $\mathrm{CP}$ sequence, a single branch was formed between TEV-TAMPS (MX_TAMPS_JX51213) and US_NW_L38714, although the length of each line indicates a considerable distance between these isolates. In this case, a separate branch was formed by US_7DA_DQ986288, US_HAT_M11458, and US_M15239. 
To see whether this phylogeny would also apply for the $\mathrm{CP}$ sequence, a similar analysis was done for this cistron. The analysis showed again that TEV-TAMPS clusters separately to the TEV isolates susceptible to RTM (Fig. 2B). In this case, a single group formed by TEV-TAMPS and the US_NW_L38714 was observed, although the distance between these isolates is con-


Fig. 3. Effect of absence or overexpression of eIF(iso)4E on TEV-TAMPS RNA accumulation. A, The TEV-TAMPS positive or $\mathbf{B}$, the negative RNA strand was quantified by real time quantitative reverse transcriptionpolymerase chain reaction in inoculated Arabidopsis thaliana plants from ecotype Col-0 wild-type (white bars), (iso) $4 E-1$ knockout (black bars), and (iso) $4 E-2$ overexpressing (gray bars) plants. For quantification, total RNA was extracted from local leaves $(0 \mathrm{~L}, 0$ days postinoculation [dpi]; $4 \mathrm{~L}, 4$ dpi) and systemic leaves ( $4 \mathrm{~S}, 4 \mathrm{dpi} ; 15 \mathrm{~S}, 15 \mathrm{dpi})$. The $e E F-1 \alpha$ mRNA was used as internal control for normalization and the $0 \mathrm{~L}$ value was considered as basal line in order to represent the rest of samples as fold of change with respect to the inoculated viral RNA. Asterisks (*) indicate the difference was statistically significant at $P<0.05$. C, Immunoblots of total protein extracts from local or systemic leaves of TEV-TAMPS inoculated plants, as described in the upper part of the figure, were performed with anti-coat protein (CP) serum to detect Tobacco etch virus (TEV) or with anti-eEF1B $\beta$ serum as loading control. siderable. For this phylogenetic tree, different Mexican isolates were not included, due to the unavailability of corresponding $\mathrm{CP}$ sequences.

eIF(iso)4E is not required for TEV-TAMPS RNA replication or stability in A. thaliana Col-0.

The interaction between eIF(iso)4E and the viral VPg has been proposed by several authors to participate in RNA stability, translation, or movement (Lellis et al. 2002; Okade et al. 2009). Additionally, eIF(iso)4E sequestration by VPg might impair translation of host mRNAs and promote viral RNA translation (Eskelin et al. 2011; Khan et al. 2008). To further understand the potential role of eIF(iso)4E in TEV-TAMPS infection of $A$. thaliana, the replication and presence of viral RNA was evaluated in local and systemic leaves of plants inoculated with semipurified viral particles obtained as described below. In addition to the knockout line (iso) $4 E-1$, an eIF(iso) $4 \mathrm{E}$ overexpressing line on Col-0 ecotype background, (iso) $4 E-2$, was included in the analysis. Accumulation of the virus positive and negative RNA strands was quantified by real time quantitative (q)RT-PCR in local leaves at 0 and 4 dpi as well as in systemic leaves at 4 and 15 dpi (Fig. 3A and B). The positive RNA level in local leaves at 0 dpi (discussed below) was employed as the reference for each line used in the experiment. It must be noted, here, that the inoculum, despite intense washing, importantly contributed to the number of virus-positive strands detected in the inoculated leaves, particularly at $0 \mathrm{dpi}$ (Fig. 3A), but not to the negative strands (Fig. 3B).

The results indicated that TEV-TAMPS accumulation in local leaves at $4 \mathrm{dpi}$ was impaired in the (iso) $4 E-1$ knockout mutant and enhanced in the (iso)4E-2-overexpressing mutant as compared with the Col-0 WT plants (Fig. 3A and B). Accumulation of the viral strands in systemic leaves at 4 and 15 dpi was not detected in the (iso) $4 E-1$ mutant plants, in contrast to WT and the (iso) $4 E-2-$ overexpressing mutant. Again, and taking together the data of positive and negative viral strands, viral accumulation in systemic leaves was enhanced in (iso)4E-2 (Fig. 3A and B). These observations indicate that eIF(iso)4E is not strictly required for the replication of TEVTAMPS RNA in local leaves, as it is in the case of TuMV and LMV inoculations (Duprat et al. 2002), but it is indispensable for the TEV-TAMPS systemic dispersion.

A. thaliana inoculation with TEV-TAMPS was not associated with visible symptoms of viral infection. Therefore, to test whether the systemic spread is able to produce viral proteins, a Western blot with commercially available TEV CP antiserum was performed on local and systemic leaves (Fig. 3C). The observed results were consistent with the behavior of positive and negative RNA strands (Fig 3A and B), and the CP level in systemic tissues was similar in the cases of Col-0 WT and (iso) $4 E-2$ plants. CP was not detected in systemic leaves of the (iso) $4 E-1$ knockout. These data further confirm that eIF(iso)4E is required for the TEV-TAMPS movement.

\section{Arabidopsis thaliana eIF(iso)4E is not required for TEV-TAMPS RNA translation.}

We evaluated the translational efficiency of TEV-TAMPS in the presence or lack of eIF(iso)4E to determine whether this particular factor is able to enhance translation of the viral RNA in planta. For this assay, inoculated A. thaliana leaves, at 4dpi, from (iso) $4 E-1$, (iso) $4 E-2$, and WT plants were used. The distribution of viral RNA was measured along sucrose density gradients (polyribosomal fractionation), using qRT-PCR of a region corresponding to the CP RNA. The mRNAs efficiently initiated for translation are found on polyribosomes (high sucrose concentration), whereas a decrease in translation initiation shows a shift towards nonpolyribosome (fractions with 
lower sucrose concentration). The profiles during fractionation were similar for WT, (iso) $4 E-1$, and (iso) $4 E$-2 plants (Fig. 4A), indicating that there were no meaningful changes on global translation levels by the absence or overexpression of eIF(iso)4E. The viral RNA was found in free RNA particles (fractions 2 to 4 ) and polyribosomal fractions with a peak of abundance between fractions 10 and 14 (Fig. 4B). This indicates that only a fraction of the total positive RNA strand is subjected to active translation in the absence, presence, or overexpression of eIF(iso)4E. The distribution of viral RNA appeared mostly unaltered in (iso) $4 E-1$ and (iso) $4 E-2$ lines as compared with WT. The distribution of a cellular mRNA used as control, the eukaryotic elongation factor $1 \alpha(e E F 1 \alpha)$ mRNA, was found mostly in heavy polysomes (Fig. 4), indicating that it is efficiently translated in the presence of the viral RNA, regardless of the eIF(iso)4E levels. The presence of viral RNA in the fractions corresponding to polysomes was dependent on ribosome association as shown by puromycin polysomedisrupting treatments (Supplementary Fig. S3). These results do not support the hypothesis that TEV-TAMPS VPg interaction with eIF(iso)4E impairs translation of endogenous transcripts or enhances its own translation, at least in the $A$. thaliana host. Moreover, it was confirmed that eIF(iso) $4 \mathrm{E}$ is not required for TEV-TAMPS translation.

\section{TEV-TAMPS CP and VPg are found}

in complexes with $A$. thaliana $\mathrm{eIF}(\mathrm{iso}) 4 \mathrm{E}$ in planta.

The eIF(iso)4E factor has been previously associated with potyvirus translation and replication (Cotton et al. 2009; Khan et al. 2006, 2008). However, in this work we obtained evidence that, in A. thaliana, the eIF(iso)4E absence or overexpression does not affect translation (association to polysomes). Moreover, eIF(iso)4E absence does not impede virus replication in local leaves. Nevertheless, eIF(iso)4E is required for the viral movement, either cell to cell or at long distance, and its systemic spread. Therefore, we asked whether eIF(iso)4E is particularly associated to viral proteins involved in the systemic spread. This interaction was tested by coimmunoprecipitation assays using protein extracts from (iso) $4 E-1$, (iso) $4 E-2$, and WT local leaves at 4 dpi. Three immunoprecipitations were performed using the antibody for $A$. thaliana eIF(iso)4E (IP-[iso]4E) or eIF4E (IP-4E) and the antibody against TEV CP. The immunoprecipitated protein complexes were separated on sodium dodecyl sulfate-polyacrylamide gel electrophoresis (SDSPAGE), were blotted, and were detected with antibodies against eIF(iso)4E, eIF4E, CP, and NIa (Fig. 5).

The Western blot against eIF(iso)4E and eIF4E in IP-(iso)4E and IP-4E demonstrated that the antibodies are able to specifically distinguish each isoform without cross-reactivity and, hence, each immunoprecipitate is expected to contain protein complexes specific for each isoform. eIF(iso) $4 \mathrm{E}$ was not detected in (iso) $4 E-1$ plants, and none of the other Western assays showed a detectable product for the IP-(iso)4E of the knockout (Fig. 5 second panel). Detection with either $\alpha$-CP or $\alpha$-NIa in the same immunoprecipitate rendered a positive signal for WT and (iso) $4 E-2$ but not in the (iso) $4 E-1$ knockout, indicating specific coimmunoprecipitation of both viral proteins in complex with eIF(iso)4E. To corroborate the specificity of this
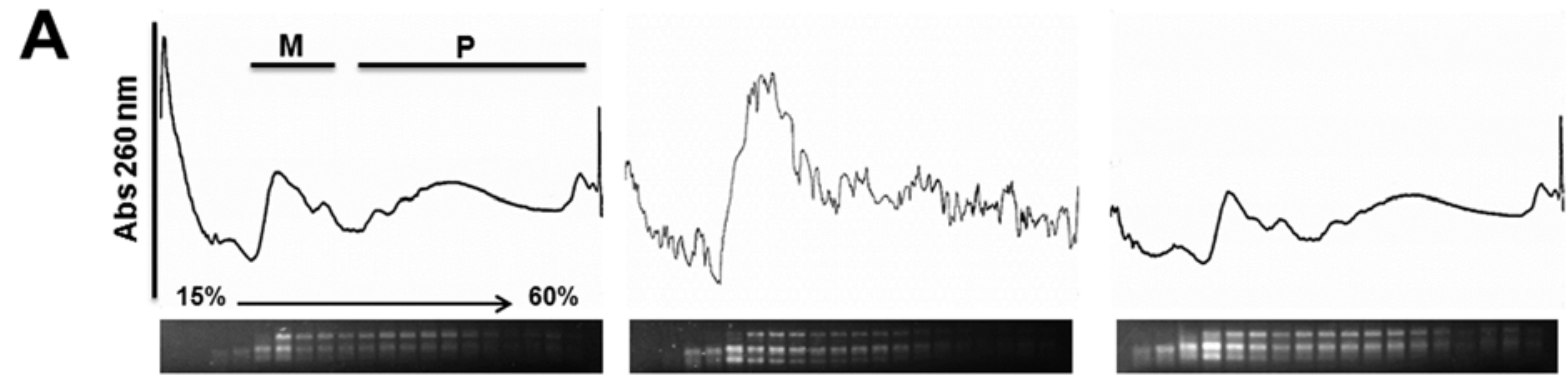

B

Col-0
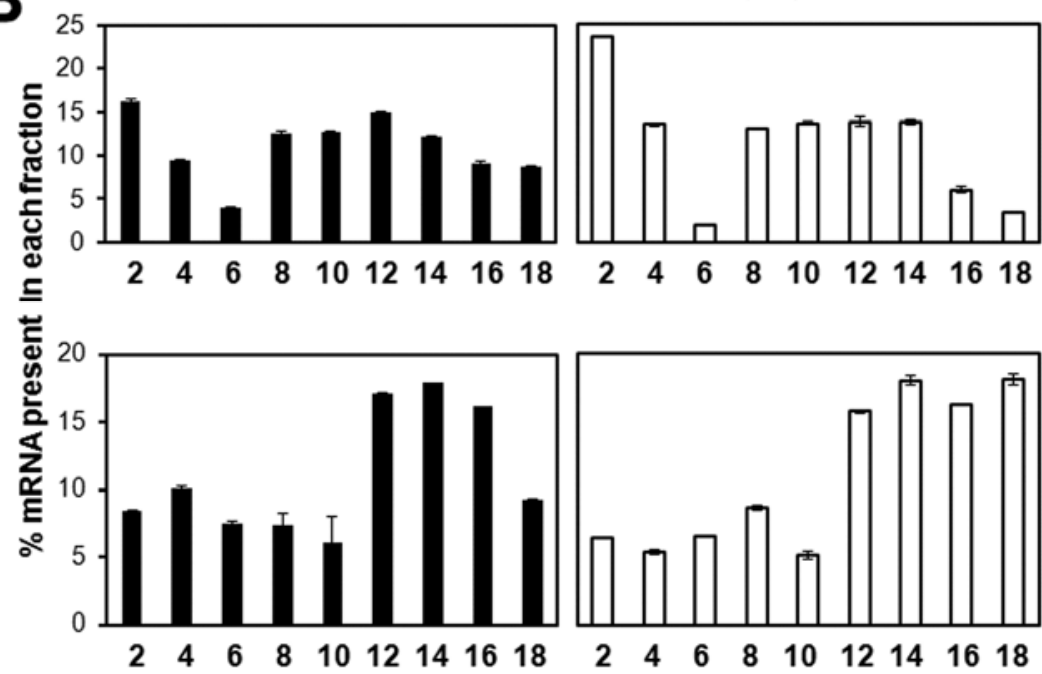

(iso) $4 E-2$


eEF1 $\alpha$

Fig. 4. Quantitation of TEV-TAMPS RNA in polysomal fractions from Arabidopsis thaliana wild-type Col-0 (black bars), (iso)4E-1 knockout (white bars), and (iso) $4 E-2$ overexpressing (gray bars) plants. A, The polysomal fractionation was performed on continuous sucrose gradients (15 to $60 \%$ ), and profiles were detected at $260 \mathrm{~nm}$. B, The RNA isolated from each fraction (pair numbers) was subjected to quantitative reverse transcription-polymerase chain reaction for the viral RNA $(C P)$, and cellular $e E F-1 \alpha$ RNA. The percentage of RNA corresponding to each sucrose fraction is shown. The distribution of viral RNA along the profile was not affected in the (iso) $4 E-1$ knockout or (iso) $4 E-2$ overexpressing mutants, similar to the behavior of the cellular mRNA in mono $(\mathrm{M})$ and polysomal $(\mathrm{P})$ pools. 
interaction, immunoprecipitation assays using $\alpha-\mathrm{CP}$ or $\alpha$ eIF4E were performed with protein extracts from 4-dpi TEVTAMPS local leaves. The CP was detected in immunoprecipitation assays of all plants, since the extract was obtained from local leaves. However, eIF(iso)4E and NIa were immunodetected only in the immunoprecipitation assays from WT and (iso) $4 E-2$ plants (Fig. 5, third panel). On the other hand, immunodetection performed with the same antibodies of IP-4E complexes returned positive for eIF4E and NIa but not $\mathrm{CP}$ and eIF(iso)4E (Fig. 5, fourth panel). These data suggest the presence of at least two different viral protein complexes, one involving $\mathrm{CP}$, NIa, and eIF(iso)4E, the other one including NIa and eIF4E. The antibody against NIa includes the portion corresponding to $\mathrm{VPg}$, and the band detected in immunoprecipitates was about $26 \mathrm{kDa}$, indicating that the protein found in these complexes is actually VPg. Therefore, TEV-TAMPS VPg is able to interact in planta with either eIF(iso)4E or eIF4E from A. thaliana, similar to reports of previous in vitro and in vivo studies with VPg from other potyviruses (Grzela et al. 2006).

\section{DISCUSSION}

Members of the translation initiation factor eIF4E family constitute a major group of host-recessive resistance genes for potyviruses (Nicaise et al. 2007; Piron et al. 2010; Robaglia and Caranta 2006; Ruffel et al. 2002). Several genetic and biochemical approaches have demonstrated that the VPg is the predominant factor mediating the eIF4E resistance and susceptibility mechanism. Based on the eIF4E function in eukaryotic translation and on the observed VPg interference of its binding to a Cap ( $\left.{ }^{7} \mathrm{mGTP}\right)$ analogue or during in vitro mRNA translation (Grzela et al. 2006; Khan et al. 2008), it has been proposed that the resistance mechanism mediated through eIF4E family members is at the level of viral translation enhancement, cellular translation inhibition, or both. However, other evidences argue against this proposal. One of these is the presence of an internal translation initiation mechanism promoted by specific regions found in the $5^{\prime}$ untranslated region of several potyviral RNAs able to efficiently support in vitro translation by directly binding other initiation factors, such as eIF4G or eIF(iso)4G, without the presence of eIF4E or eIF(iso)4E (Gallie 2001; Ray et al. 2006). The other is that inhibiting cellular translation through eIF4E sequestration by VPg, as has been demonstrated by in vitro and, recently, in vivo experiments (Eskelin et al. 2011; Miyoshi et al. 2008), would not fully explain the specificity of the virus requiring a particular eIF4E isoform depending upon the host.

In this work, we aimed to dissect the level of eIF(iso)4E participation in the infection of A. thaliana with a Mexican isolate of the TEV named TEV-TAMPS. When analyzing inoculations of TEV-TAMPS in A. thaliana Col-0 WT and eIF(iso) $4 E$ knockout plants, we noticed that this isolate reproducibly overcame the RTM mechanism only in the WT plants. TEV-TAMPS mutations in VPg and $\mathrm{CP}$, both proteins involved in the systemic movement of potyviruses, might be responsible for overcoming resistance mechanisms such as RTM and allowing the infection of $A$. thaliana. Recent publications support this idea, showing that amino acid substitutions, particularly at the $\mathrm{N}$-terminus of $\mathrm{CP}$, are determinants for the ability of LMV and PPV isolates to break the RTM resistance mechanism in A. thaliana (Decroocq et al. 2009). Furthermore, mutations in the VPg protein from TuMV have been shown to be sufficient to break the resistance imposed by $A$. thaliana eIF(iso) $4 E$ or $\operatorname{IF}($ iso $) 4 G$ knockout mutants by a mechanism independent of its interaction with these factors (Gallois et al. 2010).

The null mutation for eIF(iso)4E in A. thaliana (Duprat et al. 2002) was previously reported as resistant to both TuMV and LMV, even at local tissues. The observation that TEVTAMPS is able to overcome the RTM mechanism only in the

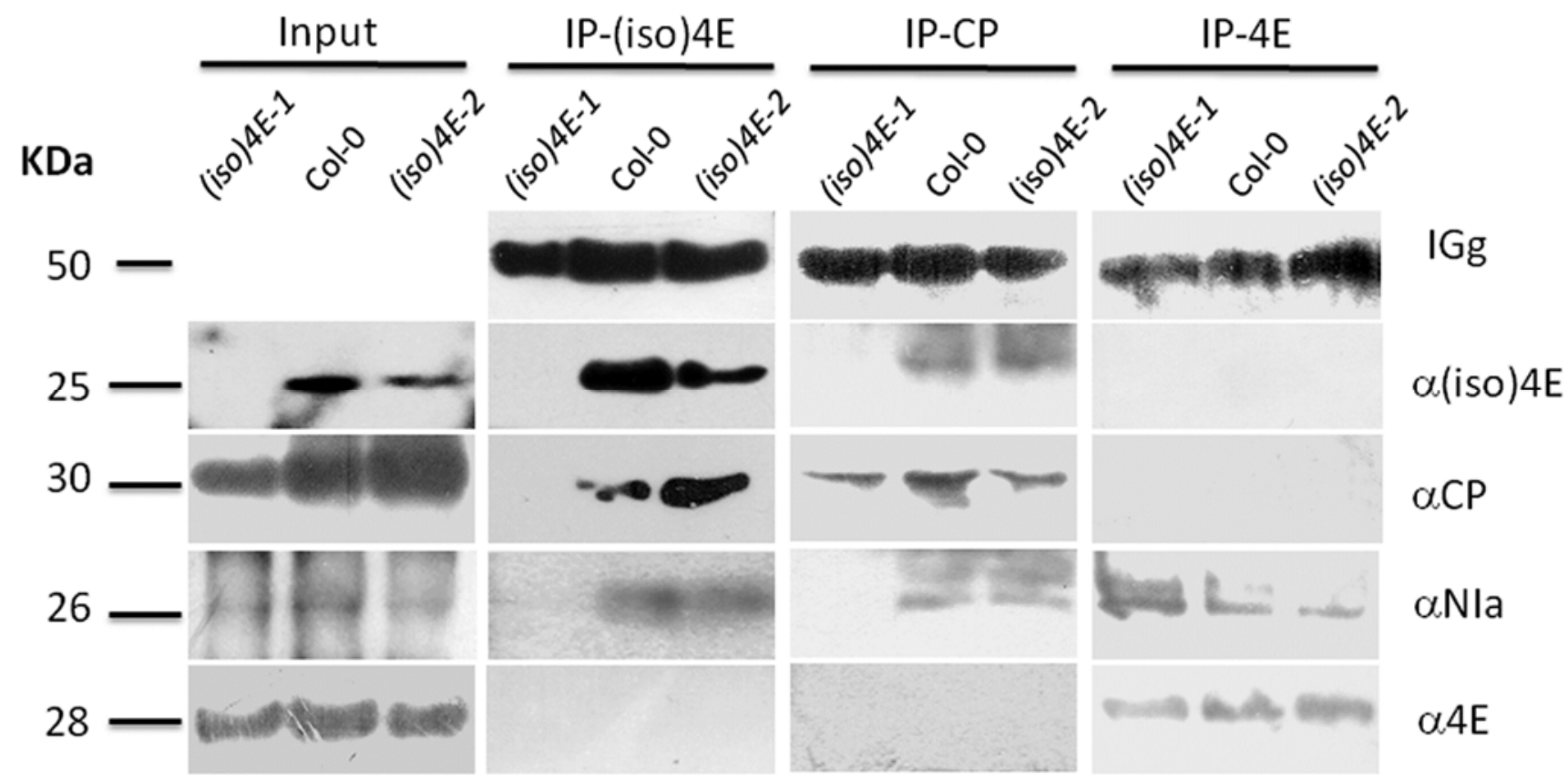

Fig. 5. Interaction of Arabidopsis thaliana Col-0 eIF(iso)4E and eIF4E with TEV-TAMPS proteins. Immunoprecipitation assays were performed using total protein extracts obtained from local leaves of A. thaliana wild-type Col-0, (iso) $4 E-1$ knockout, and (iso) $4 E$ - 2 overexpressing plants at 4 days postinoculation. Immunoprecipitates (IP) with $\alpha$-CP-TEV, $\alpha$-(iso) $4 \mathrm{E}$, and $\alpha-4 \mathrm{E}$, as well as $10 \mu \mathrm{g}$ of total protein extract (Input) were resolved with sodium dodecyl sulfatepolyacrylamide gel electrophoresis and were immunoblotted against $\alpha$-CP-TEV, $\alpha$-NIa-TEV (which also recognizes viral genome-linked protein [VPg]), $\alpha$ (iso) $4 \mathrm{E}$ and $\alpha-4 \mathrm{E}$. IP with $\alpha$-(iso) $4 \mathrm{E}$ (IP-[iso]4E) contained complexes formed by $A$. thaliana eIF(iso) $4 \mathrm{E}$ and the coat protein (CP) (30 kDa) and VPg (26 $\mathrm{kDa}$ ) viral proteins but not the $A$. thaliana eIF4E protein. Reciprocally, the IP with $\alpha-\mathrm{CP}$ (IP-CP) contained the same proteins. The IP with $\alpha-4 \mathrm{E}$ (IP-4E) did not show the presence of viral CP or Arabidopsis eIF(iso)4E but displayed a positive signal for the viral VPg (in $\alpha-\mathrm{NIa}$ ). 
presence of eIF(iso)4E but is detected in local tissues regardless the eIF(iso)4E presence allowed us to study in planta the viral translation, replication, and systemic spread. Our results indicated that abolishment of eIF(iso)4E did not impede TEVTAMPS RNA accumulation in local leaves, although overexpression of this protein positively influenced, to some extent, viral replication in both local and systemic leaves (Fig. 3). Such observation is consistent with previous findings describing the presence of eIF(iso)4E and other translational factors, such as the PolyA binding protein 2 (PABP2) and the translation elongation factor $1 \mathrm{~A}(\mathrm{eEF} 1 \mathrm{~A})$ in large vesicles associated with viral replication (Cotton et al. 2009; Hafren et al. 2010). However, TEV-TAMPS replication was not strictly dependent on the eIF(iso)4E presence, since the knockout mutant presented newly synthesized negative RNA strands in local leaves (Fig. 3B). In addition, this mutant did not show impairments in global cellular translation or in the viral RNA translation (Fig. 4), ruling out the requirement of eIF(iso)4E for these steps in the viral infective cycle, at least for the TEV-TAMPS-Arabidopsis system. Other work makes reference to two different sites within eIF4E that independently bind VPg and the Cap analogue $\mathrm{m}^{7} \mathrm{GDP}$ with similar affinity in vitro. The VPg-binding site would be different but structurally related to the CAPbinding site and reduces the mRNA Cap affinity (Michon et al. 2006). Similar interactions could occur with the TEV-TAMPS $\mathrm{VPg}$ and $\mathrm{eIF}$ (iso)4E of $A$. thaliana Col-0 but probably without a reduction in the mRNA Cap affinity, as there was not an interference with host mRNA translation.

Using co-immunoprecipitation assays, we were able to detect the TEV-TAMPS VPg in either eIF(iso) 4E or eIF4E complexes. However, we found the viral CP only in the eIF(iso) $4 \mathrm{E}$ complexes (Fig. 5). The presence of CP only in the eIF(iso)4Edependent protein complex has not been previously reported and could correlate with the requirement of this specific isoform for the viral movement in A. thaliana. Whether the interaction between eIF(iso)4E and CP is direct or is supported by other proteins within the complex remains to be solved. However, it is unlikely that VPg would recruit $\mathrm{CP}$ in the complex, since we found VPg but not CP in the eIF4E-bound complexes. Taking into account that both $\mathrm{CP}$ and $\mathrm{VPg}$ are proteins required for viral movement, we propose that particular complexes formed by eIF(iso) $4 \mathrm{E}$ and these proteins are involved in the systemic spread of TEV-TAMPS. In the absence of eIF(iso)4E, such complexes could not be formed, hence, the viral movement is restricted.

Previous reports have described the interaction between eIF(iso)4E and TuMV VPg or its precursors VPg-Pro (NIa) and $6 \mathrm{~K}-\mathrm{VPg}$-Pro (6K-NIa), suggesting a role in the disruption of cellular functions or in viral translation or replication (Beauchemin et al. 2007; Cotton et al. 2009). A. thaliana is a TuMV-susceptible plant showing severe symptoms upon infection with this potyvirus. The same plant species could be infected by TEV-TAMPS isolates without developing any obvious symptoms. For both TuMV and TEV A. thaliana susceptibility, the requirement of eIF(iso) $4 \mathrm{E}$ has been demonstrated (Duprat et al. 2002; Lellis et al. 2002). However, whereas the presence of TuMV RNA could not be detected in the inoculated leaves of an A. thaliana eIF(iso)4E knockout (Duprat et al. 2002), viral products from TEV-TAMPS could be observed in local leaves when compared with WT plants, even though the inoculated virus might account for the majority of the detected RNA. Therefore, replication of TuMV strands may be strictly dependent on eIF(iso)4E, whereas replication of TEV, although negatively affected, still occurs in the absence of eIF(iso)4E.

The analysis of CP and VPg sequences reveals that specific amino acid changes may be responsible for the ability of TEVTAMPS to overcome the RTM resistance mechanism. It will be interesting to get a further insight in the phylogeny of these proteins, when more sequences for both proteins are available from the same isolates in different geographic regions. Noticeably, TEV-TAMPS VPg interacts with both eIF(iso)4E and eIF4E in planta. However, each isoform is apparently involved in different complexes with the viral proteins, leading us to conclude that they probably exert different roles within the viral life cycle.

\section{MATERIALS AND METHODS}

\section{Plant and virus materials.}

Arabidopsis thaliana Columbia 0 (Col-0) ecotype was used as WT as well as the genetic background for the $\operatorname{eIF}(i s o) 4 E$ knockout AteIF(iso)4E-1 (Duprat et al. 2002) and the eIF(iso) $4 E$-overexpressing mutant AteIF(iso)4E-2 (MartinezSilva et al. 2012) lines. For some experiments, the ecotype Columbia 24 (C24) reported as susceptible to the TEV systemic movement was used (Mahajan et al. 1998). Individual plants were grown in pots with Metromix 200 substrate and were kept at $23^{\circ} \mathrm{C}$, under $100 \mu \mathrm{mol} \mathrm{m}{ }^{-2} \mathrm{~s}^{-1}$ light intensity, with an 8 -h light and 16-h dark photoperiod, for 6 weeks before inoculation.

TEV-TAMPS was used for inoculation of all plants. TEVTAMPS from the federal Mexican state of Tamaulipas was propagated in Nicotiana tabacum cv. Xanthi plants (6 weeks old) and were maintained under standard greenhouse conditions. The TEV-7DA isolate used in this work was expressed from a p35TEV plasmid previously reported (Bedoya and Daros 2010) and was propagated in N. tabacum cv. Xanthi plants (6 weeks old) under greenhouse conditions. TEV-TAMPS and TEV-7DA viral particles were partially purified from $N$. $t a-$ bacum symptomatic leaves, according to a procedure previously described (Gopalan 2007). The amount of viral particles was determined using ELISA quantitation with $\alpha$-CP-TEV (Agdia Inc., Elkhart, IN, U.S.A.). Six-week-old rosette leaves from $A$. thaliana plants were mechanically inoculated using a cotton swab tip with estimated similar amounts of viral particles.

\section{Total RNA isolation and TEV-TAMPS detection by final point RT-PCR.}

Total RNA from A. thaliana and $N$. tabacum plants was extracted using the Trizol method (Invitrogen Corp.; San Diego, CA, U.S.A.) and following the manufacturer's indications. The concentration was quantified by measuring absorbance at 260 $\mathrm{nm}$, and its quality was verified by electrophoresis on a $1 \%$ agarose gel. The reverse transcription reaction was performed with M-MLV reverse transcriptase (Invitrogen Corp.) according to the manufacturer's instructions, using $1 \mu \mathrm{g}$ of total RNA. Oligo dT was used as primer in the reverse transcriptase reaction. PCR assays were performed with recombinant Taq polymerase (Invitrogen Corp.) following the manufacturer's instructions, using the appropriate primers as described in Supplementary Table $\mathrm{S} 1$. The amplified products were resolved on a $1 \%$ agarose gel electrophoresis.

\section{Polysomal RNA purification and analysis.}

The separation of polysomal complexes was performed using $2 \mathrm{~g}$ of TEV-inoculated leaves, according to a previously described protocol (Davies and Abe 1995), adapted to A. thaliana (Martinez-Silva et al. 2012). After concentrating through a $60 \%$ sucrose cushion, ribosome-bound RNA was loaded on 15 to $60 \%$ sucrose gradients and was centrifuged for $2 \mathrm{~h}$ at $4^{\circ} \mathrm{C}$ in a SW-55 rotor at $250,000 \times g$. Nine fractions were separated with an Auto Densi-flow system (Labconco, Kansas City, MO, U.S.A.) coupled to a $260 \mathrm{~nm}$ absorbance detector Econo UV Monitor EM-1 (Bio-Rad, Hercules, CA, U.S.A.) and a plotter LKB BROMMA 2210 (Pharmacia LKB, Uppsala, Sweden) to 
obtain the polysomal profiles. The RNA was extracted from each fraction using phenol-chloroform. The treatment with puromycin was done by adding GTP buffer ( $20 \mathrm{mM}$ Tris- $\mathrm{HCl}$, $\mathrm{pH} 8,400 \mathrm{mM} \mathrm{KCl}, 3 \mathrm{mM} \mathrm{MgCl} 2$, and $0.5 \mathrm{mM}$ puromycin) to the pellet obtained after the $60 \%$ sucrose cushion centrifugation and incubating for $45 \mathrm{~min}$ at $37^{\circ} \mathrm{C}$. Afterwards, the extract was run through a 15 to $60 \%$ sucrose gradient.

\section{qRT-PCR.}

RNA $(2 \mu \mathrm{g})$ were treated with RQ1 DNase (Promega Corp., Madison, WI, U.S.A.) and were reverse-transcribed using MMLV-RT (Invitrogen). The qPCR was performed with qTEV primers designed with the aid of the "Primer Tools" within the Geneious program). $e E F-1 \alpha$ was used as an internal normalizing control gene. For the negative viral-strand quantitation, the $5^{\prime}$ forward oligonucleotide TEV3 was used in the reverse transcriptase reaction. The PCR amplification was performed in a 7500 DNA analyzer (Applied Biosystems, Foster City, CA, U.S.A.), using SYBR Green PCR master mix (Applied Biosystems) for signal detection. The relative viral expression was calculated after normalization with the reference gene $(e E F-1 \alpha)$, using the $2^{-\Delta \Delta \mathrm{Ct}}$ method and was represented as fold of change with respect to the inoculated RNA (either positive or negative strand) collected from the local leaves $2 \mathrm{~h}$ after inoculation. To avoid with the quantification of inoculated viral particles that did not enter the plant cells, the local leaves were extensively washed with virus-free solution and, afterwards, were subjected to RNA isolation or were further grown under standard conditions to collect local leaves at 4 dpi and systemic leaves at 4 or 15 dpi. Tukey honestly significant difference analysis $(P<$ $0.05)$ was performed on the data to estimate statistical differences between the three A. thaliana lines (WT, [iso]4E-1, and [iso]4E-2). The quantification of viral RNA distributed along polysomal fractions, obtained by sucrose gradients, was performed as described previously (Dinkova et al. 2005; MartinezSilva et al. 2012), and the percentage of the RNA in nonpolysomal and polysomal fractions was determined.

\section{Immunoblot and ELISA assays.}

Total proteins were extracted by grinding $500 \mathrm{mg}$ of leaf tissue and homogenizing it in $500 \mu \mathrm{l}$ of extraction buffer (200 $\mathrm{mM}$ Tris-HCl, pH 8.5, $50 \mathrm{mM} \mathrm{KCl,} 25 \mathrm{mM} \mathrm{MgCl} 2,2 \mathrm{mM}$ EGTA, 2\% PTE (polyoxyethylene [10] tridecyl ether), 1\% Igepal, and $5 \mu \mathrm{g}$ of cycloheximide per milliliter, $1 \mathrm{mM}$ dithiothreitol (DTT), and $1 \mathrm{mM}$ orthovanadate). After centrifugation $(12,000 \times g, 5 \mathrm{~min})$, the supernatant was recovered, and the concentration was determined by the Bradford assay (Bio$\mathrm{Rad}$ ), using bovine serum albumin as standard. After SDSPAGE, $5 \mu \mathrm{g}$ of total proteins were used directly for immunoblot analysis. The $\alpha$-eIF4E and $\alpha$-eIF(iso)4E antibodies supplied by K. S. Browning from the Department of Chemistry and Biochemistry, University of Texas at Austin, were used at 1:10,000 dilution. The $\alpha$-CP-TEV (Agdia Inc.) was used at $1: 10,000$ dilution, and the $\alpha-$ NIa antibody (from W. Dougherty) was used at 1:15,000 dilution. The antigen-antibody complexes were detected using a horseradish peroxidase-coupled goat $\alpha$ rabbit immunoglobulin $\mathrm{G}$ (IgG) according to the manufacturer's recommendations. Immunoreactive bands were detected with Immobilon Western Chemiluminescent HRP substrate (Millipore, Bedford, MA, U.S.A.).

For ELISA experiments, $100 \mu \mathrm{g}$ of total proteins obtained from uninfected or infected (15 dpi) systemic leaves of $A$. thaliana Col-0 with TEV-TAMPS or TEV-7DA were incubated, with $\alpha$-CP-TEV (Agdia) as capture antibody at 1:500 dilution. As secondary antibody, an alkaline phosphatase-coupled goat $\alpha$-rabbit IgG was used at a 1:1,000 dilution. The activity of alkaline phosphatase was indirectly estimated at $450 \mathrm{~nm}$ using
NBT (nitro-blue tetrazolium chloride) and BCIP (5-bromo-4chloro-3'-indolyphosphate p-toluidine salt) in carbonate buffer (0.1 $\left.\mathrm{M} \mathrm{NaHCO}_{3}, 1 \mathrm{mM} \mathrm{MgCl} 2, \mathrm{pH} 9.8\right)$ as substrate.

\section{Immunoprecipitation assays.}

To evaluate interactions between eIF(iso)4E and viral proteins, $3 \mathrm{~g}$ of local or systemic leaves were ground. The slurry was transferred to $15-\mathrm{ml}$ tubes with $5 \mathrm{ml}$ of extraction buffer (200 mM Tris- $\mathrm{HCl}, \mathrm{pH} 8.5,50 \mathrm{mM} \mathrm{KCl}, 25 \mathrm{mM} \mathrm{MgCl}_{2}, 2$ $\mathrm{mM}$ EGTA, heparin at $100 \mu \mathrm{g} / \mathrm{ml}, 2 \%$ PTE, $1 \%$ Igepal [previously Nonidet P40], and cycloheximide at $5 \mu \mathrm{g} / \mathrm{ml}, 1 \mathrm{mM}$ DTT, and $1 \mathrm{mM}$ orthovanadate). The extracts were vigorously mixed and were centrifuged for $15 \mathrm{~min}$ at $10,000 \times g$ at $4^{\circ} \mathrm{C}$. The upper clear supernatant was used to perform immunoprecipitation assays according to a previous report (Peritz et al. 2006), using Protein Agarose A (Millipore). The $\alpha$-eIF(iso)4E, $\alpha$-CP-TEV, and $\alpha$-eIF4E antibodies were used at 1:1,000 dilutions. The total protein extracts (Input) and the immunoprecipitates were analyzed by immunoblots.

\section{TEV-TAMPS VPg and CP sequencing and phylogenetic analysis.}

The TEV-TAMPS isolate was obtained from Capsicum annum cultivars in the county of Tampico Madero in the federal state of Tamaulipas, Mexico. The nucleotide sequences of VPg and CP were obtained after RT-PCR amplification, using specific oligonucleotides and cloning into pGEM-T Easy vector (Promega Corp.). Both sequences were cured using the BioEdit Sequence Alignment Editor, version 7.0.9.0 for Windows, and were tested against GenBank sequences using the Blast software freely accessible on the NCBI website. The sequences were manually assembled and deposited in the GenBank database with the following accession numbers: TEV-TAMPS VPg, JX512812_MX_TAMPS; TEV-TAMPS CP, JX512813_MX_TAMPS.

Available VPg and CP sequences from other TEV isolates were obtained from GenBank. Unfortunately, CP sequences were not available for all accessions used in the VPg analysis and vice versa. Nucleotide sequences for both VPg and CP cistrons were aligned using Geneious Pro 4. 8.3 created by Biomatters (available from the Biomatters Geneious Pro website) with the Clustal W algorithm (Larkin et al. 2007) and cost matrix with gap extension cost of 100 . The aligned nucleotide sequences were further translated in silico, with the same program, and were used with the Geneious tree builder with the Jukes-Cantor genetic distance model and the neighbor-joining method. A bootstrapping with a random seed of 500,000 and 1,000 replicates and no outgrouping was used. The consensus tree was selected with a threshold of 50 .

\section{ACKNOWLEDGMENTS}

This work was supported by grants to T. D. Dinkova (PAPIIT IN204309, IN210912 and CONACYT 81708) and L. Silva-Rosales (Cinvestav funds). J.-A. Daròs was funded by grant BIO2011-26741 (MEC, Spain). C. A. Contreras-Paredes and N. D. Alejandri-Ramírez were awarded with a fellowship from CONACYT for their Ph.D. studies. The authors are thankful to W. Dougherty for the NIa-TEV antibody and K. Browning (University of Texas at Austin) for Arabidopsis eIF(iso)4E and eIF4E antibodies.

\section{LITERATURE CITED}

Allison, R. F., Dougherty, W. G., Parks, T. D., Willis, L., Johnston, R. E., Kelly, M., and Armstrong, F. B. 1985. Biochemical analysis of the capsid protein gene and capsid protein of tobacco etch virus: N-terminal amino acids are located on the virion's surface. Virology 147:309-316.

Ashby, J. A., Stevenson, C. E., Jarvis, G. E., Lawson, D. M., and Maule, A. J. 2011. Structure-based mutational analysis of eIF4E in relation to 
sbml resistance to pea seed-borne mosaic virus in pea. PLoS ONE 6:e15873. Published online.

Beauchemin, C., Boutet, N., and Laliberte, J.F. 2007. Visualization of the interaction between the precursors of $\mathrm{VPg}$, the viral protein linked to the genome of Turnip mosaic virus, and the translation eukaryotic initiation factor iso 4E in Planta. J. Virol. 81:775-782.

Bedoya, L. C., and Daros, J. A. 2010. Stability of Tobacco etch virus infectious clones in plasmid vectors. Virus Res. 149:234-240.

Browning, K. S. 2004. Plant translation initiation factors: It is not easy to be green. Biochem. Soc. Trans. 32:589-591.

Carrington, J. C., and Whitham, S.A . 1998. Viral invasion and host defense: Strategies and counter-strategies. Curr. Opin. Plant Biol. 1:336-341.

Cosson, P., Sofer, L., Hien Le, Q., Leger, V., Schurdi-Levraud, V., Whitham, S. A., Yamamoto, M. L., Gopalan, S., Le Gall, O., Candresse, T., Carrington, J. C., and Revers, F. 2010. RTM3, which controls longdistance movement of potyviruses, is a member of a new plant gene family encoding a Meprin and TRAF Homology domain-containing protein. Plant Physiol. 154:222-232.

Cosson, P., Schurdi-Levraud, V., Le, Q. H., Sicard, O., Caballero, M. Roux, F., Le Gall, O., Candresse, T., and Revers, F. 2012. The RTM resistance to potyviruses in Arabidopsis thaliana: Natural variation of the $R T M$ genes and evidence for the implication of additional genes. PLoS ONE 6:e39169. Published online.

Cotton, S., Grangeon, R., Thivierge, K., Mathieu, I., Ide, C., Wei, T. Y., Wang, A. M., and Laliberte, J. F. 2009. Turnip mosaic virus RNA replication complex vesicles are mobile, align with microfilaments, and are each derived from a single viral genome. J. Virol. 83:10460-10471.

Chisholm, S. T., Mahajan, S. K., Whitham, S. A., Yamamoto, M. L., and Carrington, J. C. 2000. Cloning of the Arabidopsis RTM1 gene, which controls restriction of long-distance movement of Tobacco etch virus. Proc. Natl. Acad. Sci. U.S.A. 97:489-494.

Chisholm, S. T., Parra, M. A., Anderberg, R. J., and Carrington, J. C. 2001. Arabidopsis RTM1 and RTM2 genes function in phloem to restrict longdistance movement of Tobacco etch virus. Plant Physiol. 127:1667-1675.

Chu, M., Johnson, M., Thornbury, D., Black, L., and Pirone, T. 1995. Nucleotide sequence of a strain of Tobacco etch virus that does not cause Tabasco pepper wilt. Virus Genes 10:283-288.

Chung, B.Y ., Miller, W. A., Atkins, J. F., and Firth, A. E. 2008. An overlapping essential gene in the Potyviridae. Proc. Natl. Acad. Sci. U.S.A. 105:5897-5902

Davies, E., and Abe, S. 1995. Methods for isolation and analysis of polyribosomes. Methods Cell Biol. 50:209-222.

Decroocq, V., Sicard, O., Alamillo, J. M., Lansac, M., Eyquard, J. P., Garcia, J. A., Candresse, T., Le Gall, O., and Revers, F. 2006. Multiple resistance traits control Plum pox virus infection in Arabidopsis thaliana. Mol. Plant-Microbe Interact. 19:541-549.

Decroocq, V., Salvador, B., Sicard, O., Glasa, M., Cosson, P., SvanellaDumas, L., Revers, F., Garcia, J.A., and Candresse, T. 2009. The determinant of potyvirus ability to overcome the RTM resistance of Arabidopsis thaliana maps to the N-terminal region of the coat protein. Mol. Plant-Microbe Interact. 22:1302-1311.

Dinkova, T. D., Aguilar, R., and Sanchez de Jimenez, E. 2000. Expression of maize eukaryotic initiation factor (eIF) iso4E is regulated at the translational level. Biochem. J. 351:825-831.

Dinkova, T. D., Keiper, B. D., Korneeva, N. L., Aamodt, E. J., and Rhoads, R. E. 2005. Translation of a small subset of Caenorhabditis elegans mRNAs is dependent on a specific eukaryotic translation initiation factor 4E isoform. Mol. Cell Biol. 25:100-113.

Dinkova, T. D., Marquez-Velazquez, N. A., Aguilar, R., Lázaro-Mixteco, P. E., and Sanchez de Jimenez, E. 2011. Tight translational control by the initiation factors eIF4E and eIF(iso)4E is required for maize seed germination. Seed Sci, Res, 21:85-93.

Dolja, V. V., Haldeman-Cahill, R., Montgomery, A. E., Vandenbosch, K. A., and Carrington, J. C. 1995. Capsid protein determinants involved in cell-to-cell and long distance movement of tobacco etch potyvirus. Virology 206:1007-1016.

Duprat, A., Caranta, C., Revers, F., Menand, B., Browning, K. S., and Robaglia, C. 2002. The Arabidopsis eukaryotic initiation factor (iso)4E is dispensable for plant growth but required for susceptibility to potyviruses. Plant J. 32:927-934.

Eskelin, K., Hafren, A., Rantalainen, K.I., and Makinen, K. 2011. Potyviral VPg enhances viral RNA translation and inhibits reporter mRNA translation in planta. J. Virol. 85:9210-9221.

Gallie, D. R. 2001. Cap-independent translation conferred by the $5^{\prime}$ leader of Tobacco etch virus is eukaryotic initiation factor $4 \mathrm{G}$ dependent. J. Virol. 75:12141-12152.

Gallois, J. L., Charron, C., Sanchez, F., Pagny, G., Houvenaghel, M. C., Moretti, A., Ponz, F., Revers, F., Caranta, C., and German-Retana, S. 2010. Single amino acid changes in the turnip mosaic virus viral ge- nome-linked protein (VPg) confer virulence towards Arabidopsis thaliana mutants knocked out for eukaryotic initiation factors eIF(iso)4E and eIF(iso)4G. J. Gen. Virol. 91:288-293.

Gao, Z., Johansen, E., Eyers, S., Thomas, C. L., Noel Ellis, T. H., and Maule, A. J. 2004. The potyvirus recessive resistance gene, $s b m 1$, identifies a novel role for translation initiation factor eIF4E in cell-to-cell trafficking. Plant J. 40:376-385.

Gopalan, S. 2007. A multidirectional non-cell autonomous control and a genetic interaction restricting tobacco etch virus susceptibility in Arabidopsis. PLoS ONE 2:e985. Published online.

Grzela, R., Strokovska, L., Andrieu, J. P., Dublet, B., Zagorski, W., and Chroboczek, J. 2006. Potyvirus terminal protein VPg, effector of host eukaryotic initiation factor eIF4E. Biochimie 88:887-896.

Hafren, A., Hofius, D., Ronnholm, G., Sonnewald, U., and Makinen, K. 2010. HSP70 and its cochaperone CPIP promote potyvirus infection in Nicotiana benthamiana by regulating viral coat protein functions. Plant Cell 22:523-535.

Hwang, J., Li, J., Liu, W. Y., An, S. J., Cho, H., Her, N. H., Yeam, I., Kim, D., and Kang, B. C. 2009. Double mutations in eIF4E and eIFiso4E confer recessive resistance to Chilli veinal mottle virus in pepper. Mol. Cells 27:329-336.

Jackson, R. J., Hellen, C. U., and Pestova, T. V. 2010. The mechanism of eukaryotic translation initiation and principles of its regulation. Nat. Rev. Mol. Cell Biol. 11:113-127.

Jenner, C. E., Nellist, C. F., Barker, G .C., and Walsh, J. A. 2010. Turnip mosaic virus (TuMV) is able to use alleles of both eIF4E and eIF(iso)4E from multiple loci of the diploid Brassica rapa. Mol. PlantMicrobe Interact. 23:1498-1505.

Kang, B. C., Yeam, I., Frantz, J. D., Murphy, J. F., and Jahn, M. M. 2005 The pvrl locus in Capsicum encodes a translation initiation factor eIF4E that interacts with Tobacco etch virus VPg. Plant J. 42:392-405.

Khan, M. A., Miyoshi, H., Ray, S., Natsuaki, T., Suehiro, N., and Goss, D. J. 2006. Interaction of genome-linked protein (VPg) of Turnip mosaic virus with wheat germ translation initiation factors eIFiso4E and eIFiso4F. J. Biol. Chem. 281:28002-28010.

Khan, M. A., Miyoshi, H., Gallie, D. R., and Goss, D. J. 2008. Potyvirus genome-linked protein, $\mathrm{VPg}$, directly affects wheat germ in vitro translation: Interactions with translation initiation factors eIF4F and eIFiso4F. J. Biol. Chem. 283:1340-1349.

Larkin, M. A., Blackshields, G., Brown, N. P., Chenna, R., McGettigan, P. A., McWilliam, H., Valentin, F., Wallace, I. M., Wilm, A., Lopez, R. Thompson, J. D., Gibson, T. J., and Higgins, D. G. 2007. Clustal W and Clustal X version 2.0. Bioinformatics. 23:2947-2948.

Lellis, A. D., Kasschau, K. D., Whitham, S. A., and Carrington, J. C. 2002. Loss-of-susceptibility mutants of Arabidopsis thaliana reveal an essential role for eIF(iso)4E during potyvirus infection. Curr. Biol. 12:1046-1051.

Leonard, S., Plante, D., Wittmann, S., Daigneault, N., Fortin, M. G., and Laliberte, J. F. 2000. Complex formation between potyvirus VPg and translation eukaryotic initiation factor 4E correlates with virus infectivity. J. Virol. 74:7730-7737.

Mahajan, S. K., Chisholm, S. T., Whitham, S. A., and Carrington, J. C. 1998. Identification and characterization of a locus (RTM1) that restricts long-distance movement of Tobacco etch virus in Arabidopsis thaliana. Plant J. 14:177-186.

Martinez-Silva, A. V., Aguirre-Martinez, C., Flores-Tinoco, C. E., AlejandriRamirez, N. D., and Dinkova, T. D. 2012. Translation initiation factor AteIF(iso)4E is involved in selective mRNA translation in Arabidopsis thaliana seedlings. PLoS One 7:e31606. Published online.

Michon, T., Estevez, Y., Walter, J., German-Retana, S., and Le Gall, O. 2006. The potyviral virus genome-linked protein VPg forms a ternary complex with the eukaryotic initiation factors eIF4E and eIF4G and reduces eIF4E affinity for a mRNA Cap analogue. FEBS (Fed. Eur. Biochem. Soc.) Lett. 273:1312-1322.

Miyoshi, H., Okade, H., Muto, S., Suehiro, N., Nakashima, H., Tomoo, K., and Natsuaki, T. 2008. Turnip mosaic virus VPg interacts with Arabidopsis thaliana $\mathrm{eIF}(\mathrm{iso}) 4 \mathrm{E}$ and inhibits in vitro translation. Biochimie 90:1427-1434.

Naderpour, M., Lund, O. S., Larsen, R., and Johansen, E. 2010. Potyviral resistance derived from cultivars of Phaseolus vulgaris carrying $b c-3$ is associated with the homozygotic presence of a mutated eIF4E allele. Mol. Plant Pathol. 11:255-263.

Nicaise, V., German-Retana, S., Sanjuan, R., Dubrana, M. P., Mazier, M. Maisonneuve, B., Candresse, T., Caranta, C., and LeGall, O. 2003. The eukaryotic translation initiation factor $4 \mathrm{E}$ controls lettuce susceptibility to the potyvirus Lettuce mosaic virus. Plant Physiol. 132:1272-1282.

Nicaise, V., Gallois, J. L., Chafiai, F., Allen, L. M., Schurdi-Levraud, V., Browning, K. S., Candresse, T., Caranta, C., Le Gall, O., and GermanRetana, S. 2007. Coordinated and selective recruitment of eIF4E and eIF4G factors for potyvirus infection in Arabidopsis thaliana. FEBS (Fed. Eur. Biochem. Soc.) Lett. 581:1041-1046. 
Okade, H., Fujita, Y., Miyamoto, S., Tomoo, K., Muto, S., Miyoshi, H., Natsuaki, T., Rhoads, R. E., and Ishida, T. 2009. Turnip mosaic virus genome-linked protein VPg binds C-terminal region of cap-bound initiation factor $4 \mathrm{E}$ Orthologue without exhibiting host cellular specificity. J. Biochem. 145:299-307.

Peritz, T., Zeng, F., Kannanayakal, T. J., Kilk, K., Eiriksdottir, E., Langel, U., and Eberwine, J. 2006. Immunoprecipitation of mRNA-protein complexes. Nat. Protoc. 1:577-580.

Piron, F., Nicolai, M., Minoia, S., Piednoir, E., Moretti, A., Salgues, A., Zamir, D., Caranta, C., and Bendahmane, A. 2010. An induced mutation in tomato eIF4E leads to immunity to two potyviruses. PLoS ONE 5:e11313. Published online.

Rajamaki, M.-L., and Valkonen, J. P. 1999. The 6K2 protein and the VPg of potato virus A are determinants of systemic infection in Nicandra physaloides. Mol. Plant-Microbe Interact. 12:1074-1081.

Ray, S., Yumak, H., Domashevskiy, A., Khan, M. A., Gallie, D. R., and Goss, D. J. 2006. Tobacco etch virus mRNA preferentially binds wheat germ eukaryotic initiation factor (eIF) 4G rather than eIFiso4G. J. Biol. Chem. 281:35826-35834.

Robaglia, C., and Caranta, C. 2006. Translation initiation factors: A weak link in plant RNA virus infection. Trends Plant Sci. 11:40-45.

Rodriguez, C. M., Freire, M. A., Camilleri, C., and Robaglia, C. 1998. The Arabidopsis thaliana cDNAs coding for eIF4E and eIF(iso)4E are not functionally equivalent for yeast complementation and are differentially expressed during plant development. Plant J. 13:465-473.

Roudet-Tavert, G., Michon, T., Walter, J., Delaunay, T., Redondo, E., and Le Gall, O. 2007. Central domain of a potyvirus VPg is involved in the interaction with the host translation initiation factor eIF4E and the viral protein HcPro. J. Gen. Virol. 88:1029-1033.

Ruffel, S., Dussault, M. H., Palloix, A., Moury, B., Bendahmane, A., Robaglia, C., and Caranta, C. 2002. A natural recessive resistance gene against potato virus $\mathrm{Y}$ in pepper corresponds to the eukaryotic initiation factor 4E (eIF4E). Plant J. 32:1067-1075.

Ruffel, S., Gallois, J. L., Lesage, M. L., and Caranta, C. 2005. The recessive potyvirus resistance gene pot- 1 is the tomato orthologue of the pepper pvr2-eIF4E gene. Mol. Genet. Genomics 274:346-353.
Ruud, K. A., Kuhlow, C., Goss, D. J., and Browning, K. S. 1998. Identification and characterization of a novel cap-binding protein from Arabidopsis thaliana. J. Biol. Chem. 273:10325-10330.

Rybicki, E. P., and Shukla, D. D. 1992. Coat protein phylogeny and systematics of potyviruses. Arch. Virol. Suppl. 5:139-170.

Sato, M., Nakahara, K., Yoshii, M., Ishikawa, M., and Uyeda, I. 2005. Selective involvement of members of the eukaryotic initiation factor $4 \mathrm{E}$ family in the infection of Arabidopsis thaliana by potyviruses. FEBS (Fed. Eur. Biochem. Soc.) Lett. 579:1167-1171.

Silva-Rosales, L., Becerra-Leor, N., Ruiz-Castro, S., Teliz-Ortiz, D., and Noa-Carrazana, J. C. 2000. Coat protein sequence comparisons of three Medican isolates of papaya ringspot virus with other geographical isolates reveal a close relationship to American and Australian isolates. Arch. Virol. 145:835-843.

Taliansky, M., Torrance, L., and Kalinina, N. O. 2008. Role of plant virus movement proteins. Method Mol. Biol. 451:33-54.

Urcuqui-Inchima, S., Haenni, A.L., and Bernardi, F. 2001. Potyvirus proteins: A wealth of functions. Virus Res. 74:157-175.

Wei, T., Zhang, C., Hong, J., Xiong, R., Kasschau, K. D., Zhou, X., Carrington, J. C., and Wang, A. 2010. Formation of complexes at plasmodesmata for potyvirus intercellular movement is mediated by the viral protein P3N-PIPO. PLoS Pathog 6:e1000962. Published online.

Whitham, S. A., Yamamoto, M. L., and Carrington, J. C. 1999. Selectable viruses and altered susceptibility mutants in Arabidopsis thaliana. Proc. Natl. Acad. Sci. U.S.A. 96:772-777.

Whitham, S. A., Anderberg, R. J., Chisholm, S. T., and Carrington, J. C. 2000. Arabidopsis RTM2 gene is necessary for specific restriction of Tobacco etch virus and encodes an unusual small heat shock-like protein. Plant Cell 12:569-582.

\section{AUTHOR-RECOMMENDED INTERNET RESOURCES}

Biomatters Geneious Pro website: www.geneious.com

NCBI Blast software: blast.ncbi.nlm.nih.gov/

NCBI GenBank: www.ncbi.nih.gov 